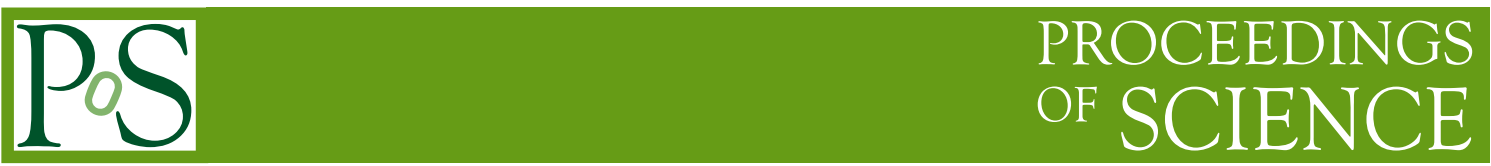

\title{
KM3NeT/ARCA sensitivity to neutrino point sources
}

\section{Agata Trovato ${ }^{a}$ and Javier Barrios-Martí ${ }^{* b}$ for the KM3NeT Collaboration}

${ }^{a}$ INFN-LNS, via S. Sofia 62, 95123 Catania, Italy

Email:atrovato@lns.infn.it

${ }^{b}$ Instituto de Física Corpuscular, IFIC (UV-CSIC), Parque Científico, C/Catedrático José Beltrán 2, E-46980 Paterna, Spain

Email:jabamareific.uv.es

\begin{abstract}
KM3NeT is a large research infrastructure that will consist of a network of deep-sea neutrino telescopes in the Mediterranean Sea, one of which, the KM3NeT/ARCA detector installed in the CapoPassero site (Italy), is optimised for studying high-energy neutrinos of cosmic origin. Thanks to its geographical location on the Northern hemisphere, KM3NeT/ARCA can observe most of the Galactic Plane, including the Galactic Centre. Under the hypothesis of hadronic gamma emission and transparent sources, models for galactic neutrino sources are well constrained by TeV gammaray observations and allow to obtain realistic perspectives. Sensitivities for galactic sources such as the supernova remnant RXJ1713.7-3946 and the pulsar wind nebula Vela X are presented as well as sensitivities for a generic point source with an $E^{-2}$ spectrum, which represents an approximation for the spectrum of extragalactic candidate neutrino sources.
\end{abstract}

The 34th International Cosmic Ray Conference,

30 July- 6 August, 2015

The Hague, The Netherlands

${ }^{*}$ Speaker. 


\section{Introduction}

The recent discovery by the IceCube collaboration of a high-energy neutrino flux of extraterrestrial origin [1] has greatly enhanced the interest in the field of Neutrino Astronomy. Unambiguous identification of the emitting neutrino sources will require neutrino telescopes with good angular resolution and full sky coverage. KM3NeT is a multi- $\mathrm{km}^{3}$ high-energy neutrino telescope to be installed in the Mediterranean Sea. The IceCube discovery and the $\theta_{13}$ measurement motivated the collaboration to propose KM3NeT-2.0 consisting of two detectors with different granularity, KM3NeT/ARCA [2] and KM3NeT/ORCA [3] (Astroparticle and Oscillation Research in the Abyss). The former is dedicated to high-energy neutrino astronomy and will be installed at the KM3NeT-It site in Capo Passero (Italy), the latter aims at studying the neutrino mass hierarchy and will be installed at the KM3NeT-Fr site offshore Toulon (France).

Neutrinos are detected by measuring the Cherenkov light induced by charged secondary particles emerging from a neutrino interaction. KM3NeT will consist of arrays of light detectors constructed in the deep, clear water of the Mediterranean Sea that serves both as target material and Cherenkov radiator and shield for atmospheric neutrinos. The light detectors are photo-multiplier tubes (PMTs) arranged in glass spheres that resist the water pressure (digital optical modules, DOM [4]). In KM3NeT, the optical modules will, for the first time in neutrino optical Cherenkov telescopes, house multiple small PMTs instead of a large one, optimising the photo-cathode area per optical module. The optical modules are arranged along flexible strings with a total height of about $700 \mathrm{~m}$ for KM3NeT/ARCA. This detector will consist of two building blocks of 115 strings each, with 18 DOMs per string and a spacing of $36 \mathrm{~m}$ between DOMs. The footprint will be roughly circular with an average distance between strings of about $90 \mathrm{~m}$. Each block will have a volume of about $\sim 0.5 \mathrm{~km}^{3}$, so KM3NeT/ARCA will have approximately the same volume of IceCube. An extension to 6 building blocks of KM3NeT/ARCA is envisaged to fully exploit the scientific potential of the detector especially in the search of Galactic point sources.

Due to its location in the Northern hemisphere, ARCA will be complementary to the IceCube field of view and overlap it to a large extent: the same sources will be observed at different energies, sources that are not visible to IceCube can be observed, and, due to the reduced light scattering in water as compared to ice, the neutrino direction will be measured with much better precision. The expected KM3NeT/ARCA sensitivity will allow for the detection of the reported IceCube flux [1] within less than one year of observation [5].

The operation of gamma-ray telescopes have revealed in the last decades a large number of $\mathrm{TeV} \gamma$-ray sources in our Galaxy associated with Supernova Remnants (SNR) and Pulsar Wind Nebulae (PWD). Assuming a hadronic scenario for the $\gamma$-ray production and transparent sources, a corresponding neutrino flux can be calculated allowing to estimate the detection perspective of neutrino telescopes.

In the following sections the detection perspective of KM3NeT for point sources will be discussed, with particular emphasis to Galactic sources.

\section{Simulation codes and analysis}

The software used in this work has been developed by the ANTARES Collaboration [6] and 
adapted to $\mathrm{km}^{3}$-scale detectors. The code provides a full simulation for neutrinos with energy in the range $10^{2} \div 10^{8} \mathrm{GeV}$, including their interaction in the medium and the propagation of the emerging secondary particles, the light generation and propagation in water and the detector response. The depth and the optical water properties measured at the KM3NeT-It Capo Passero site are used [7]. Background light due to the presence of ${ }^{40} \mathrm{~K}$ in salt water has been also simulated.

The neutrino induced events can be divided into two classes, track-like and cascade-like events, each class requiring a specific event reconstruction. The track-like events are due to CC $v_{\mu}$ and $\bar{v}_{\mu}$ interactions that, for $\mathrm{E}_{v}>\mathrm{TeV}$, produce in the final state muons with track lengths of the order of kilometers and trajectories almost collinear with the parent neutrino direction. Also $v_{\tau}\left(\bar{v}_{\tau}\right)$ CC interactions can produce a muon in the final state when the emerging $\tau(\bar{\tau})$ decays in a $\mu(\bar{\mu})(\mathrm{BR} \sim$ $17 \%)$. The reconstruction algorithm used for track-like events is described in [8] and it is based on the timing information of the hits and on their position. It permits to reach a resolution, calculated as the median angle between the reconstructed track and the generated neutrino track, of about $0.2^{\circ}$ at $10 \mathrm{TeV}$ and an energy resolution of $\sim 0.28$ in $\log \left(\mathrm{E}_{\mu}\right)$ for events with $E_{v}$ above $10 \mathrm{TeV}$. The cascade-like topology is originated by $v_{e}\left(\bar{v}_{e}\right)$ CC interactions, $v_{\tau}\left(\bar{v}_{\tau}\right)$ CC interactions when the emerging $\tau(\bar{\tau})$ produces a shower in the final state $(\mathrm{BR} \sim 83 \%)$ and all NC neutrino interactions. The $v_{\tau}$ CC events where a cascade is seen both at the tau creation and decay are called "double bang" events but, due to the short $\tau$ lifetime compared to the size of each shower, this signature is only expected to be detectable for $v_{\tau}$ with energy $E \gtrsim \mathrm{PeV}$. The direction reconstruction for cascade-like events is based on the principle that the amount of light emitted under the Cherenkov angle with respect to the shower axis is greater than in the other directions [9]. For high energy cascades $\left(E_{v}>50 \mathrm{TeV}\right)$ taking place within the instrumented volume, a median angular resolution of less than $2^{\circ}$ and an energy resolution of roughly $10 \%$ are reached.

In point sources searches, the simulated events are analysed through statistical techniques to look for an excess around the source position. Indeed, the neutrino signal from a cosmic source needs to be identified among the large background of atmospheric muons and neutrinos, both produced by the interaction of primary cosmic rays with the atmosphere. The atmospheric neutrino event rate is calculated assuming the Honda et al. [10] conventional atmospheric model, the Enberg at al. [11] prompt component and adding the correction due to the cosmic rays knee on the neutrino spectrum as described in [12]. Atmospheric muons are simulated using the MUPAGE event generator [13]. In the analysis presented here two simulated muon event samples are used, one with muon bundle energies exceeding $10 \mathrm{TeV}$, corresponding to a live-time of about 3 months, the other with energies exceeding $50 \mathrm{TeV}$ equivalent to $\sim 7$ months of live-time.

The optimisation of the signal to background ratio is dependent on the source features (e.g. energy spectrum, angular extension). Atmospheric neutrinos are an unavoidable background but a key for a possible discrimination against cosmic neutrinos is their different spectrum, which is softer then the expected spectrum of cosmic neutrinos. Atmospheric muons represents a significant background for the search of cosmic neutrinos having track-like signatures but can be rejected looking at upward-going events, which can be only produced by neutrinos, the only particles that can traverse the whole Earth without being absorbed.

The techniques employed for the point source searches using the cascade and the track events are different. In fact, even if the cascade angular resolution is worst with respect to that of the track events, the cascade analysis permits to survey the upper part of the sky that usually is excluded for 
the track point source analysis due to the huge background of atmospheric muons. At the moment the cascade analysis is not fully completed. Only the results of the track analysis will be reported here.

The discovery potential, that is the signal flux required to obtain an observation at a given significance level (e. g. $5 \sigma$ or $3 \sigma$ ) with $50 \%$ probability [14], has been taken as a figure of merit of the telescope performance. The method used to calculate the discovery potential is an "unbinned" method [15]. In this case two hypotheses are considered: $H_{b k g}$ and $H_{b k g+s i g} . H_{b k g}$ is the hypothesis that data consist only of background while $H_{b k g+s i g}$ is the hypothesis that signal events are presents in the data in addition to the background. To test the compatibility of the data with this two different hypotheses a test static is defined as the ratio

$$
L R=\log \frac{\prod_{i=1}^{n} P\left(x_{i} \mid H_{\text {sig }+b k g}\right)}{\prod_{i=1}^{n} P\left(x_{i} \mid H_{b k g}\right)}=\sum_{i=1}^{n} \log \frac{P\left(x_{i} \mid H_{\text {sig }+b k g}\right)}{P\left(x_{i} \mid H_{b k g}\right)}
$$

where $P\left(x_{i} \mid H_{\text {sig }+b k g}\right)$ and $P\left(x_{i} \mid H_{b k g}\right)$ are the probabilities that the event $i$ occurs under the hypothesis $H_{s i g+b k g}$ or $H_{b k g}, x_{i}$ is the value of a given variable $x$ used to describe the event and $n$ is the total number of recorded events in a given period of time. This method requires Probability Density Functions (PDF) that describe the distributions signal and background events as a function of $x$, $P D F_{\text {sig }}(x)$ and $P D F_{b k g}(x)$. In this way the eq. 2.1 can be written as:

$$
L R=\sum_{i=1}^{n} \log \frac{\frac{n_{\text {sig }}}{n} \times P D F_{\text {sig }}\left(x_{i}\right)+\left(1-\frac{n_{\text {sig }}}{n}\right) \times P D F_{b k g}\left(x_{i}\right)}{P D F_{b k g}\left(x_{i}\right)}
$$

where $n_{\text {sig }}$ is the expected number of signal events in the sample of $n$ events.

In the analysis of track-like events, a Boosted Decision Tree (BDT) from the ROOT TMVA package [16] is used. The distributions of the BDT output for the signal and background events are used as PDFs, so in this case $x_{i}$ in eq. 2.2 is the BDT value for the event $i$. The BDT uses, for each event: a set of variables chosen to describe the quality of each step of the muon direction reconstruction algorithm, the final reconstructed zenith angle, declination, right ascension, distance of the reconstructed track from the source position $\alpha$, reconstructed track length and vertex position, number of hits selected in the reconstruction procedure.

Only atmospheric neutrinos are included in the BDT training as background since they represent the main background in this analysis, while the atmospheric muon rejection is obtained with a cut on the reconstructed zenith angle, on $\alpha<10^{\circ}$ and on the reduced likelihood $\Lambda$ given by the direction reconstruction algorithm. The cut on the zenith angle corresponds to about $10^{\circ}$ above the horizon and is optimised for each particular source considering that each source can reach a different elevation depending on its declination. Fig. 1 shows, on the left, the distribution of the generated and reconstructed zenith angle $\theta$ for the atmospheric muons. In the reference system of these simulations, events with $\theta<90^{\circ}$ are upgoing while events with $\theta>90^{\circ}$ are downgoing. At a depth of $3500 \mathrm{~m}$, where the detector will be located, a depletion of the muon flux is expected allowing to look a few degrees above the horizon. Only atmospheric muons generated with $\theta_{\mu}$ greater than about $97^{\circ}$ reach the detector but the reconstructed zenith angle $\theta_{\text {rec }}$ can be smaller. Therefore, atmospheric muons mis-reconstructed as upgoing still represent a non neglegible background. Cuts on $\alpha, \theta$ and $\Lambda$ are needed to reduce the atmospheric muon background to a negligible level. The effect of these cuts, optimised for the analysis of RXJ1713.7-3946, is shown in Fig. 1. 


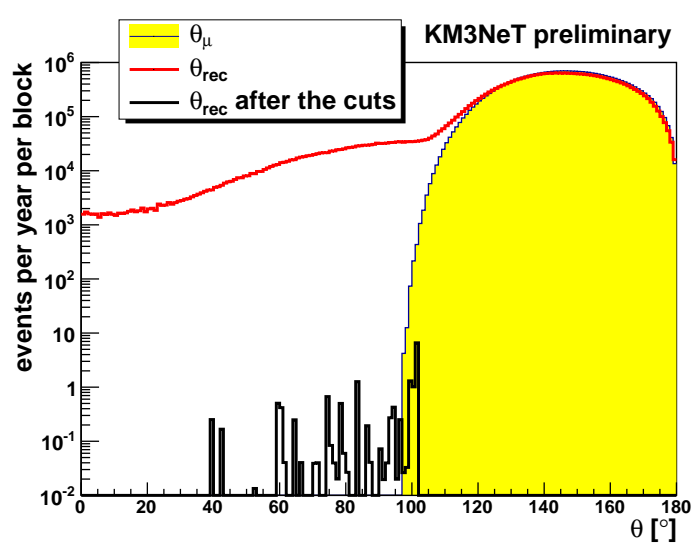

Figure 1: Zenith angle $\theta$ of atmospheric muons at generation (yellow area) and reconstruction level (red line). The black line shows reconstructed $\theta$ of the events remaining after the cuts of the RXJ1713.7-3946 analysis.

\section{Results}

Amongst the galactic objects, SNRs are probably the most promising neutrino sources. In particular, the SNR RXJ1713.7-3946 and the PWN Vela X are at present two of the most intense galactic objects in the high-energy gamma-ray band and are used here to evaluate the KM3NeT/ARCA performance. In both cases the expected neutrino spectra are calculated from the measured $\gamma$-ray spectrum under the hypotheses of a transparent source and $100 \%$ hadronic emission. The discovery flux is also evaluated for a generic $E^{-2}$ point source, as a possible approximation for neutrino extragalactic sources.

The young shell-type SNR RX J1713.7-3946 has been observed by H.E.S.S. in several campaigns [17] and its energy gamma spectrum is measured up to about $100 \mathrm{TeV}$. The source has large intensity and a relatively large size with a complex morphology. Moreover, having a declination of $-39^{\circ} 46^{\prime}$ it is visible by $\mathrm{KM} 3 \mathrm{NeT}$ for about $80 \%$ of the time. It is therefore a good reference case. This SNR has been simulated as an extended homogeneous neutrino source with a $0.6^{\circ}$ radius and an energy spectrum parametrised as [18]:

$$
\Phi(E)=16.8 \times 10^{-15}\left[\frac{E}{\mathrm{TeV}}\right]^{-1.72} e^{-\sqrt{E / 2.1 T e V}} \mathrm{GeV}^{-1} \mathrm{~s}^{-1} \mathrm{~cm}^{-2}
$$

The left panel of Fig. 2 shows the neutrino energy distributions for different neutrino flavor CC interactions simulated from this source. The $v_{\tau}+\bar{v}_{\tau}$ that have tracks $(\mu)$ in the final state are shown separately w.r.t. the case when the $\tau(\bar{\tau})$ decay produces a shower (indicated with "sh" in the legend). Only events reconstructed up to $12^{\circ}$ above the horizon and with $\alpha<1^{\circ}$ are considered in this plot. The reconstructed zenith cut $\theta<102^{\circ}$ is used in the analysis of this source. This is a compromise between the maximum elevation reached by the source (about $14^{\circ}$ above the horizon) and the contribution of the atmospheric muon background. The channels that have showers in the final state give only a small contribution to the signal and for this reason have been neglected in all the analyses described here. For the same reason the NC interactions have not been considered. The right plot of Fig. 2 shows the output of the BDT for this analysis. The BDT distributions are normalized to 1 in order to be used as input PDF for the unbinned procedure described in the previous section.

Vela $\mathrm{X}$ is one of the nearest pulsar wind nebulae (PWN) and it is associated with the energetic Vela pulsar PSR B0833-45. Even if PWNs are generally treated as leptonic sources ( $\gamma$-ray emitters 


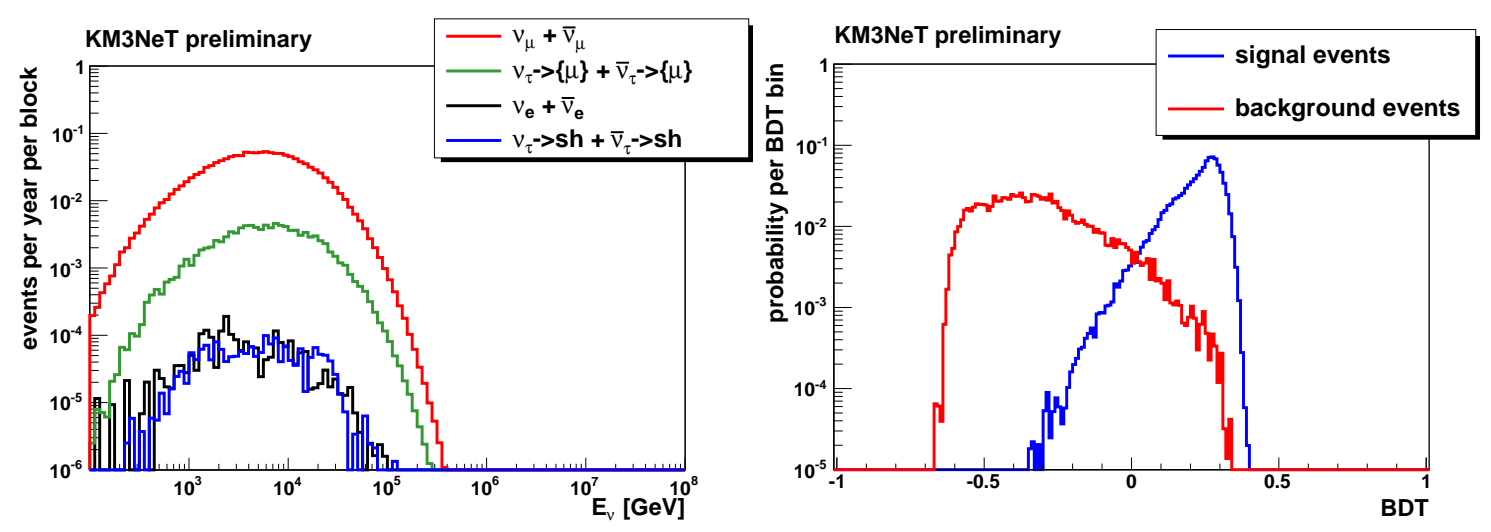

Figure 2: Left: Energy distributions of $v+\bar{v}$ expected from $\mathrm{RX} \mathrm{J1713.7-3946} \mathrm{with} \mathrm{the} \mathrm{cuts} \theta<102^{\circ}$ and $\alpha<1^{\circ}$. Right: BDT distributions for background (including atmospheric neutrinos and muons) and signal events for the RX J1713.7-3946 analysis.

through inverse Compton), interpretation of $\mathrm{TeV} \gamma$-ray emission from Vela $\mathrm{X}$ in terms of hadronic interaction is discussed by some authors (see i.e. [19, 20]). The first VHE $\gamma$-ray emission from Vela X was reported by the H.E.S.S. Collaboration [21]. It has recently been updated [22] with data from the 2005-2007 and 2008-2009 observation campaigns and using a more accurate method for the background subtraction. The new data are characterised by a higher gamma flux and a harder energy spectrum. From the differential energy spectrum extracted from an integration radius of $0.8^{\circ}$ around the centre at $\mathrm{RA}=08^{h} 35^{m}, \mathrm{DEC}=-45^{\circ} 36^{\prime}$, the corresponding neutrino emission spectrum has been derived using the Vissani prescription [23]. The neutrino spectrum is parametrised as:

$$
\Phi(E)=7.2 \times 10^{-15}\left[\frac{E}{\mathrm{TeV}}\right]^{-1.36} e^{-(E / 7 \mathrm{TeV})} \mathrm{GeV}^{-1} \mathrm{~s}^{-1} \mathrm{~cm}^{-2}
$$

Also in this case the source extension has been simulated as a flat spatial distribution within a disk of $0.8^{\circ}$ radius.

Under the assumed hypotheses, the significance of the observation as a function of the observation time for KM3NeT/ARCA and the full KM3NeT ( 6 building blocks) detectors has been calculated and is shown in Fig. 3. An observation with $3 \sigma$ significance is expected with the KM3NeT/ARCA detector after about 2.5 and 4 years for Vela X and RXJ1713.7-3946, respectively. The extension of KM3NeT permits to reach a $5 \sigma$ significance of the observation after a reasonable amount of time (about 2.5 year for Vela X and 4 years for RXJ1713.7-3946). The major uncertainty in this calculation is due to the contribution of conventional component of the neutrino flux. This uncertainty, shown as a shaded area in fig. 3, is taken into account assuming a $\pm 25 \%$ variation in the normalization of the Honda et al. flux model [10].

The $5 \sigma$ discovery flux $\Phi_{5 \sigma}$ has been also calculated for a generic point source with an $E^{-2}$ spectrum as a function of the declination (Fig. 4). Only events with reconstructed zenith angle up to $10^{\circ}$ above the horizon $\left(\theta<100^{\circ}\right)$ have been considered for declination lower than $50^{\circ}$. For higher declination it's needed to extend the zenith angle cut otherwise KM3NeT has no visibility. In particular the discovery flux for last point at declination $+70^{\circ}$ is evaluated with a cut $\theta<107^{\circ}$. The 3 years observation time has been chosen in such a way to have a comparable exposure with 

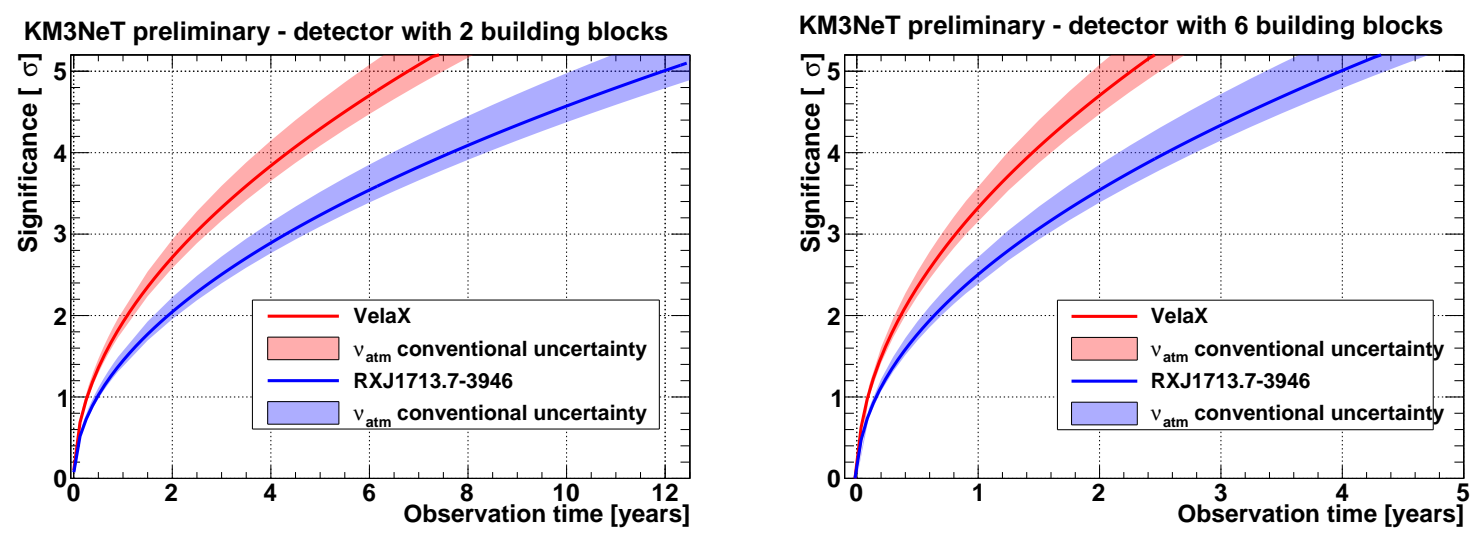

Figure 3: Significance of the RXJ1713.7-3946 and Vela X observation as a function of observation time for the KM3NeT/ARCA detector (left) and full KM3NeT detector with 6 building blocks (right). The shaded areas show the effect of the uncertainties on the conventional component of the atmospheric neutrino flux.

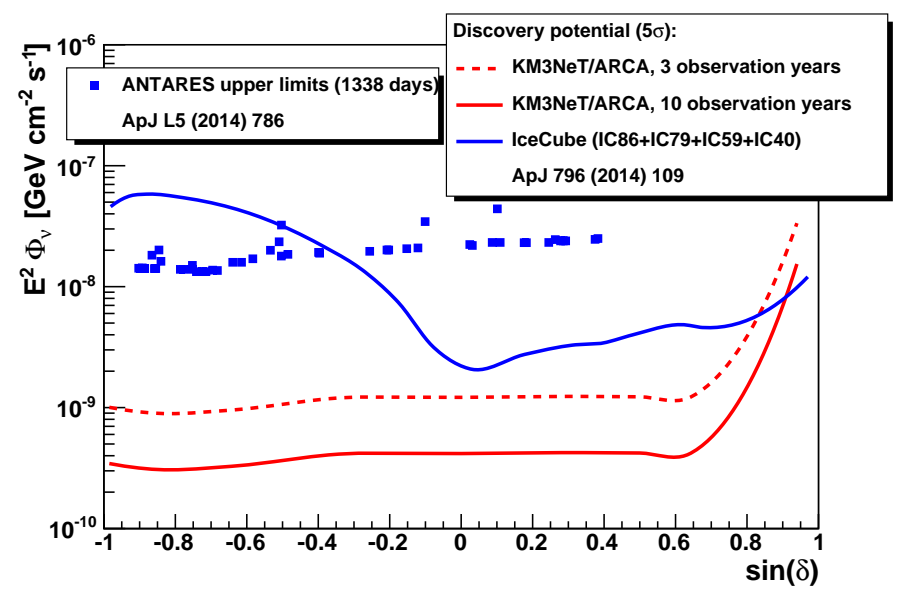

Figure 4: KM3NeT/ARCA $5 \sigma$ discovery potential per flavor for point sources emitting a neutrino flux with a $E^{-2}$ spectrum, for 3 and 10 years of data taking. The flux values are shown as function of the source declination. For comparison the corresponding one flavor discovery potential to sources with an $E^{-2}$ spectrum for the IceCube detector [24] and the upper limits quoted by ANTARES [25] are also shown.

respect to the IceCube results in [24]. The different trend of the KM3NeT and IceCube curves is mainly due to the geographical location of the detectors.

\section{Conclusions and perspectives}

The results presented in this paper represent the status of the point source analysis and show that at least the most intense galactic ones are at reach for KM3NeT. In particular,the expectation for RXJ1713.7-3946 and Vela X are reported. The inclusion in the simulation of a realistic source morphology extrapolated from the high energy $\gamma$-ray maps measured by H.E.S.S. is in progress. This will provide a more realistic description of the spatial extension of the sources. The estimate of discovery potential for other galactic sources, as well as a staking analysis of several candidate galactic sources, will be investigated in the near future.

The discovery potential for point sources with $E^{-2}$ spectrum shows that KM3NeT has a very large field of view thus not only complementing, but also overlapping to a large extent the IceCube one. KM3NeT can thus provide important contributions to the new born field of neutrino astronomy. 


\section{References}

[1] M. G. Aartsen et al. (IceCube Collaboration), Phys. Rev. Lett. 113 (2014) 101101.

[2] P. Piattelli et al. (KM3NeT Collaboration), these proceedings $(I D=1158)$.

[3] B. Juergen et al. (KM3NeT Collaboration), these proceedings $(I D=1140)$.

[4] R. Bruijn et al. (KM3NeT Collaboration), these proceedings (ID=1157).

[5] D. Stransky et al. (KM3NeT Collaboration), these proceedings (ID=1107).

[6] D. Bailey, Ph.D. Thesis, available on http://antares.in2p3.fr/Publications/index.html

[7] G. Riccobene et al., Astropart. Phys. 27 (2006) 1.

[8] A. Trovato et al. (KM3NeT Collaboration), these proceedings $(I D=1114)$.

[9] D. Stransky et al. (KM3NeT Collaboration), these proceedings (ID=1108).

[10] M. Honda, T. Kajita, K. Kasahara, S. Midorikawa, and T. Sanuki, Phys. Rev. D 75 (2007) 043006.

[11] R. Enberg, M. H. Reno, and I. Sarcevic, Phys. Rev. D 78 (2008) 043005.

[12] M. G. Aartsen et al. (IceCube Collaboration), Phys. Rev. D 89 (2014) 062007.

[13] Y. Becherini et al., Astropart. Phys. 25 (2006) 1.

[14] G.C. Hill et al., World Scientific eProceedings, Statistical problems in particle physics, astrophysics and cosmology (2005) 108.

[15] J. Braun et al., Astropart. Phys. 29 (2008) 299.

[16] A. Hoecker et al., PoS ACAT 040 (2007) [arXiv: physics / 0703039 ].

[17] F. Aharonian et al. (H.E.S.S. Collaboration), Astronomy \& Astrophysics 464 (2007) 235-243; Corrigendum in Astronomy \& Astrophysics 531 (2011) C1.

[18] S.R. Kelner et al., Phys. Rev. D 74 (2006) 034018.

[19] D. Horns at al. Astronomy \& Astrophysics 451 (2006) L1.

[20] L. Zhang and X. C. Yang 2009 ApJ 699 (2009) L153.

[21] F. Abramowski et al. (H.E.S.S. Collaboration), Astronomy \& Astrophysics 448 (2006) L43.

[22] F. Aharonian et al., Astronomy \& Astrophysics 548 (2012) A38.

[23] F. L. Villante and F. Vissani, Phys. Rev. D 78 (2008) 103007; F. Vissani and F.L. Villante, NIM A 588 (2008) 123 and F. Vissani, Astr.Ph. 26 (2006) 310.

[24] M. G. Aartsen et al. (IceCube Collaboration), Astroph. J. 796 (2014) 109.

[25] S. Adrián-Martínez et al. (Antares Collaboration), Astroph. J. Lett. L5 (2014) 786. 